\title{
Políticas públicas y salud en materia de inmigración: Puntos críticos en la accesibilidad del sistema público chileno
}

\author{
Carla Frías Ortega \\ Académica UnIVERSIDAD de ChILE \\ carlafrias@med.uchile.cl \\ Caterine Galaz Valderrama \\ ACAdÉMICA UnIVERSIDAD DE CHILE \\ cgalazvalderrama@uchile.cl \\ Rolando Poblete Melis \\ Académico Universidad Central de Chile \\ rpobletem@ucentral.cl
}

Resumen: A partir de una caracterización de población inmigrante realizada en cuatro regiones de Chile, se analiza el perfil de las personas inmigradas y sus niveles de acceso al sistema de salud. A partir de este diagnóstico, se presentan las principales problemáticas que afecta a la población migrante en cuanto a acceso al sistema público. 
Se visualiza como la imposibilidad de acceder al sistema privado de salud vigente, al no cumplir los requisitos exigidos y la condición migratoria de algunos sectores, ha hecho aumentar proporcionalmente el acceso a la salud pública de este colectivo. La metodología del estudio consistió en un análisis mixto: cuantitativo (a partir del estudio de datos secundarios de la encuesta Casen 2013 y la ex Ficha de Protección Social del gobierno chileno) y cualitativo (desarrollo de cuatro focus group y 45 entrevistas en cuatro regiones de este país). Los resultados indican que las barreras de inclusión al sistema de salud de inmigrantes son estructurales, sociales y personales. Asimismo los programas sanitarios no poseen mecanismos de divulgación específicos orientados a este colectivo, lo que les obstaculiza el acceso por falta de información y no sólo por barreras administrativas y legales. La estructura de sistema chileno de salud al ser de carácter mixto y combinar lo público-privado, centra el acceso a través del pago (ya sea por cotizaciones o por pago directo) lo que implica que las personas deben estar trabajando o disponer de las condiciones económicas mínimas para poder solventarlo. Si bien existe una cobertura básica que cubre a las personas extranjeras, los prejuicios del funcionariado de salud obstaculizan su acceso. También se constató que el temor de la población inmigrante en situación de ingreso irregular al país limita su asistencia al sistema sanitario por temor a la deportación.

Palabras clave: Inmigración, salud, políticas públicas, accesibilidad, discriminación.

\section{Public Policies and Health in terms of Immigration: Critical Points in the Accessibility of the Chilean Public Health System.}

Abstract: Taking a characterisation of the immigrant population from four regions of Chile as a starting point, the profile of the immigrants is analysed along with their levels of access to the health system. From this appraisal, the main problems affecting the migrant population regarding access to the public health system are presented. The impossibility of gaining access to the current private health system for this group on account of failing to meet the necessary requirements, along with the migratory nature of some sectors, is seen to lead to a proportionate increase in the numbers of these people seeking access to the public health system. The methodology used in this study consisted of a mixed approach: quantitative analysis (based on the study of secondary data extracted from the Casen 2013 survey and the Chilean Government's former Social Protection 
Statistics) and qualitative analysis (the development of four focus groups and 45 interviews in four regions of the country). The results indicate that the barriers preventing immigrants from being included in the health system are structural, social and personal. At the same time, healthcare programmes do not have any specific mechanisms for divulging information to this group, which hinders such access due to a lack of information rather than being solely attributable to administrative and legal barriers. The fact that the Chilean health system has a mixed structure, combining the private with the public sector, focuses access on the ability to pay (either through contributions or by direct payment), which implies that people should be working or have the minimum financial wherewithal to be able to afford it. Even though there is a basic scheme that covers foreigners, the prejudices of the public health employees hinder their access. It was also seen that the fear of the immigrant population in a position of irregular entry in the country limits their attending the health system because of fear of deportation.

Keywords: Immigration, health, public policies, accessibility, discrimination. 



\section{Políticas públicas y salud en materia de inmigración: puntos críticos en la accesibilidad del sistema público chileno}

Carla

Frías Ortega

Caterine Galaz Valderrama

Rolando Poblete Melis

Recibido: 02/06/2016

Aceptado: 21/02/2017

\section{AGRADECIMIENTOS}

Las reflexiones que sustentan este artículo han contado con el apoyo de la Subsecretaría de Evaluación Social del Ministerio de Desarrollo Social (ID: 730566-23-LE14, 2014-2015) y de la Vicerrectoría de Investigación y Desarrollo, VID, de la Universidad de Chile (U- Inicia, Galaz, 2015-2016).

\section{ANTECEDENTES: INCIDENCIA DEL MODELO DE ESTADO EN LOS SISTEMAS DE SALUD}

Para facilitar la comprensión de las diferencias que presenta la comunidad inmigrante en Chile en relación a los sistemas de salud europeos, se requiere tener como antecedente que según la Organización Mundial de la Salud (OMS), "un sistema de salud es la suma de todas las organizaciones, instituciones y recursos cuyo objetivo principal consiste en mejorar la salud. Un sistema de salud necesita personal, financiación, información, suministros, transportes y comunicaciones, así como una orientación y una dirección generales. Además tiene que proporcionar buenos tratamientos y servicios que respondan a las necesidades de la población y sean justos desde el punto de vista financiero." 
(Organización Mundial de la Salud, 2016). Se entiende, entonces, como el conjunto de las organizaciones, instituciones y recursos dedicados a la actividad sanitaria, lo que involucra a los servicios de salud personales, los servicios de salud pública o el desempeño de una actividad intersectorial. En este trabajo nos centraremos en una de estas tres dimensiones señaladas: la accesibilidad a los servicios de salud pública.

No se debe olvidar que existen diversos debates de como clasificar los sistemas de salud ya sea basados en enfoques conceptuales o bien metodológicos. Gómez-camelo (2005) distingue entre diferentes características para la clasificación los sistemas de salud: oferta y demanda, naturaleza de los bienes (público, privado o la mezcla de ambos), financiamiento (recaudo fiscal; cotizaciones; y pagos privados a seguros y gastos de bolsillo) y organización y estructura (Gómez-camelo, 2005). Si miramos los sistemas de salud europeos (Mladovsky, 2007), vemos que los sistemas de salud siguen dos grandes patrones: uno en torno a sistemas nacionales de salud financiados a través de los impuestos (modelo Beveridge) y otro basado en sistemas de seguridad social financiados por cotizaciones sociales obligatorias (modelo Bismarck). En ambos casos la financiación es eminentemente pública. Veremos más adelante, que en el caso chileno, el sistema es mixto.

A continuación se presenta una tabla resumen con los principales componentes a tener cuenta en el caso de los sistemas de salud europeos (Mladovsky, 2007). 


\section{Tabla I: Comparación de sistema de salud vigentes en países de la Unión Europea}

\begin{tabular}{|c|c|c|c|c|c|c|c|}
\hline & $\begin{array}{l}\text { Tipo } \\
\text { Siste- } \\
\text { ma }\end{array}$ & $\begin{array}{c}\text { Cartera de } \\
\text { servicios } \\
\text { definida } \\
\text { a nivel } \\
\text { central }\end{array}$ & $\begin{array}{l}\text { Modo } \\
\text { de pro- } \\
\text { visión } \\
\text { predom. } \\
\text { en AP }\end{array}$ & $\begin{array}{c}\text { \% de } \\
\text { Provisión } \\
\text { pública en } \\
\text { Hospitales }\end{array}$ & $\begin{array}{l}\text { Liber- } \\
\text { tad } \\
\text { elec- } \\
\text { ción } \\
\text { médico } \\
\text { AP }\end{array}$ & $\begin{array}{c}\text { Filtro } \\
\text { para } \\
\text { acceso } \\
\text { a espe- } \\
\text { cialis- } \\
\text { tas }\end{array}$ & $\begin{array}{l}\text { Listas } \\
\text { de } \\
\text { espera } \\
\text { como } \\
\text { pro- } \\
\text { blema } \\
\text { político }\end{array}$ \\
\hline Dinamarca & SNS & NO & Privada & 97 & Limitado & SI & SI \\
\hline España & SNS & SI (positiva) & Pública & 75 & Limitado & SI & SI \\
\hline Finlandia & SNS & NO & Pública & 89 & Limitado & SI & SI \\
\hline Irlanda & SNS & NO & Privada & 100 & SI & $\begin{array}{l}\text { Parcial- } \\
\text { men te }\end{array}$ & SI \\
\hline Italia & SNS & SI(positiva) & Pública & 81 & SI & SI & SI \\
\hline Portugal & SNS & NO & Pública & 86 & Limitado & SI & SI \\
\hline Reino Unido & SNS & SI(negativa) & Privada & 96 & SI & SI & SI \\
\hline Suecia & SNS & NO & Pública & 98 & Limitado & NO & SI \\
\hline Alemania & SSS & SI(negativa) & Privada & 49 & SI & SI & NO \\
\hline Austria & SSS & NO & Privada & 72 & $\begin{array}{c}\text { SI } \\
\text { (incen- } \\
\text { tivos } \\
\text { para no } \\
\text { cambiar) }\end{array}$ & NO & NO \\
\hline Bélgica & SSS & SI(positiva) & Privada & 34 & $\begin{array}{c}\text { SI } \\
\text { (incen- } \\
\text { tivos } \\
\text { para no } \\
\text { cambiar) }\end{array}$ & NO & NO \\
\hline Francia & SSS & SI(positiva) & Privada & 66 & $\mathrm{SI}$ & NO & NO \\
\hline Grecia & SSS & NO & Pública & 69 & $\begin{array}{c}\text { SI } \\
\text { (incen- } \\
\text { tivos } \\
\text { para no } \\
\text { cambiar) }\end{array}$ & NO & SI \\
\hline Luxemburgo & SSS & SI(positiva) & Privada & 68 & SI & NO & NO \\
\hline $\begin{array}{l}\text { Países } \\
\text { Bajos }\end{array}$ & SSS & SI(positiva) & Privada & 0 & SI & SI & SI \\
\hline
\end{tabular}

Fuente: Health in Transition (OMS). OECD Health Working Papers No. 50: Health Systems Institutional Characteristics: a survey of 29 OECD countries. 
En el caso de estos sistemas de salud si bien en general se plantea el acceso a las prestaciones de salud de las población inmigrante residente en el país, es relevante plantear que en los últimos años, se ha producido una distancia entre lo que la normativa establece y los accesos efectivos de este colectivo a dichos servicios sanitarios. Por ejemplo, en Alemania, por ley se establece que debe ser prestada la atención sanitaria a toda la población incluida la inmigrante, no obstante su ejecución queda en manos de los organismos municipales (la visita médica requiere de autorización de asuntos sociales; o en algunas zonas han optado por otorgar una tarjeta de seguridad sanitaria a refugiados para reducir las demoras y evitar obstáculos en el acceso). En Italia, mientras tanto, toda persona (nacional o inmigrante) debe inscribirse en el Servicio Sanitario Nacional. Con la tarjeta sanitaria se acceder a un médico de base, servicios de urgencias y las hospitalizaciones. En este país se debe disponer de la residencia, por tanto quienes no estén en condición regular deben solicitar la tarjeta S.T.P. (extranjero temporalmente presente) donde el requisito es no tener recursos económicos. En Francia, las personas en situación irregular acceden a cuidados médicos con tarjeta de Ayuda Médica, esta cubre asistencia médica y hospitalaria, en consultas o servicios que no superen las tarifas reembolsadas por la Seguridad Social, aunque no cubre exámenes, ni medicamentos, etc. En Suiza, las personas en situación irregular tienen derecho a salud, lo que está garantizado en la Constitución federal de Suiza, aunque si supera los tres meses de residencia deben obligatoriamente afiliarse a un seguro que es altamente costoso. En tanto, en Portugal tienen acceso al Servicio Nacional de Salud en igualdad de condiciones que la población nacional los/ las inmigrantes residentes. Los inmigrantes en situación irregular acceden a la sanidad pero al no ser contribuyentes deben pagar el costo total de prestaciones. Lo mismo sucede en el Reino Unido. La mención de estos casos permite ver los puntos críticos a nivel europeo y las fisuras que se visualizan en el acceso de la población inmigrante.

En tanto, a diferencia de los estados europeos, Chile presenta características particulares tanto de su modelo de Estado como de sus modelos de salud. Para considerar una lectura del acceso al sistema de salud chileno, se requiere tener en cuenta dos hechos concretos: el cambio que se materializó en dictadura de 
un estado protector a un estado neoliberal; y además que en ese marco, la red pública de salud comenzó a tener un carácter mixto: compuesta por entidades públicas pero también privadas. En el marco de la dictadura militar, en 1979 y bajo la impronta de una lógica neoliberal, se genera un cambio importante en el sistema: por un lado, se transfirió a las municipalidades parte de la administración y la Atención Primaria, lo que la hizo depender de los niveles de ingresos de cada zona geográfica (produciéndose desigualdades territoriales), pero por otro, se creó FONASA como seguro básico público mientras se incentivó la privatización del mercado de aseguramiento de la salud en instituciones de salud previsional (ISAPRE). Así, el sistema público se financia con aportes fiscales y con la cotización en salud de las personas afiliadas, que se concentran en los quintiles de menor ingreso económico y atiende a un porcentaje mayor de población nacional. Por otro lado, las ISAPRES agrupan a menos población y con más recursos socioeconómicos. De esta manera, esta reforma incidió en la acentuación de la segmentación del sistema y las inequidades entre diferentes colectivos sociales.

\section{ANTECEDENTES GENERALES SOBRE MOVIMIENTOS MIGRATO- RIOS EN CHILE.}

En los últimos veinte años, Chile se ha vuelto un horizonte de instalación para personas de otros países. Estos movimientos trasnacionales, se han acentuado a partir de la década de los 90, recibiendo especialmente personas provenientes de Centro y Sudamérica (Perú, Argentina, Bolivia, Colombia, Ecuador, República Dominicana y Haití, entre otros). Pese a que comienza a situarse como una problemática de atención social, según la Encuesta de Caracterización Económica Nacional (CASEN, 2013), el porcentaje de población inmigrada no superaría el $2,8 \%$ del total de la población (aunque se desconoce el número de población inmigrada en situación irregular que reside en el país). No obstante, el fenómeno comienza a tener cabida dentro de las políticas públicas, ante la evidencia de la progresión de este movimiento trasnacional. 


\section{Gráfico 1. Número total de inmigrantes en Chile (2008-2013)}

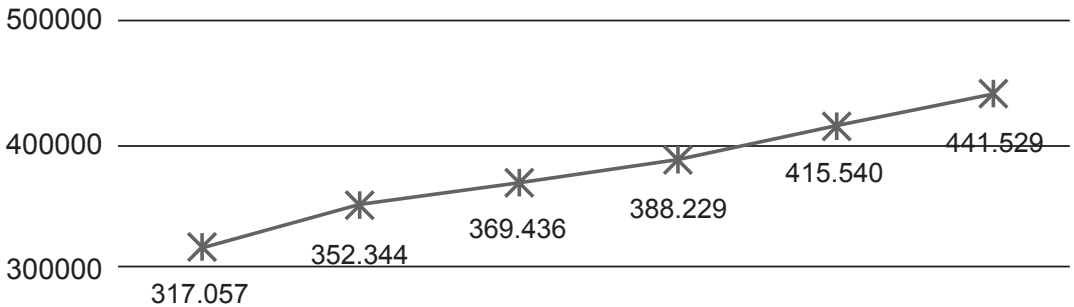

Fuente: elaboración propia en base DEM (2014)

Considerando el perfil de personas que migran a Chile, vemos a través del análisis desarrollado en la investigación que casi el $80 \%$ proviene de América Latina (Casen, 2013) y en su mayoría se sitúan en grandes centros urbanos como Santiago, Valparaíso, Antofagasta, Arica, Valparaíso y Punta Arenas. No obstante, tal como sucede con la población nacional, la concentración mayor se da en la capital con un $67,8 \%$ de inmigrados/as. Asimismo, como sucede a nivel mundial, existe una tendencia a la feminización de la población inmigrada: existe un predominio de mujeres, alcanzando un 55\% de la población residente. Dentro del conjunto de población inmigrada, especialmente de algunas procedencias como República Dominicana y Colombia, se evidencia que las mujeres cada vez más emigran solas o son pioneras en el proyecto migratorio como parte de estrategias familiares para luego desarrollar procesos de reagrupación familiar (Galaz, 2016). Asimismo, las características de los colectivos que migran a Chile, suelen tender a ser personas en edad económicamente activa, más del $64 \%$ de los/las inmigrados/as se ubican entre los 15 y 44 años. Los resultados del análisis secundario muestra que un aspecto importante a tener en cuenta es que el promedio de escolaridad en la población inmigrada supera a la nacida en Chile: en los mayores de 15 años nacidos en Chile es de 10,7 años, 
mientras que en los grupos inmigrados es de 12,6 años (Casen, 2013). Sólo en el colectivo boliviano baja a una media de 10,6 años (lo que plantea algunos desafíos para las regiones del norte de Chile donde principalmente reside este colectivo). En cuanto al acceso al área de salud fue importante visualizar las condiciones de los sujetos inmigrados y su relación con el sistema de salud vigente en el país. Tal como sucede con la población nacional, la mayoría de esta población accede a prestaciones a través del Fondo Nacional de Salud (FONASA) ${ }^{1}$, aunque existen indicios de aumento en el acceso a cobertura de salud privada a través del sistema de Isapres ${ }^{2}$, a medida que avanza su proceso de regularización y permanencia en el país. No obstante, existe un sector de la población inmigrada que no accede a ningún tipo de prestación de salud (un 8,9\% de esta población, sin considerar la población irregular). Este porcentaje de población es motivo de preocupación precisamente por los riesgos de vulnerabilidad que comporta estar fuera de una cobertura sanitaria.

\section{Gráfico 2. Distribución de la población nacida en Chile e inmigrante según sistema de salud al que accede (periodo 2009 y 2013)}

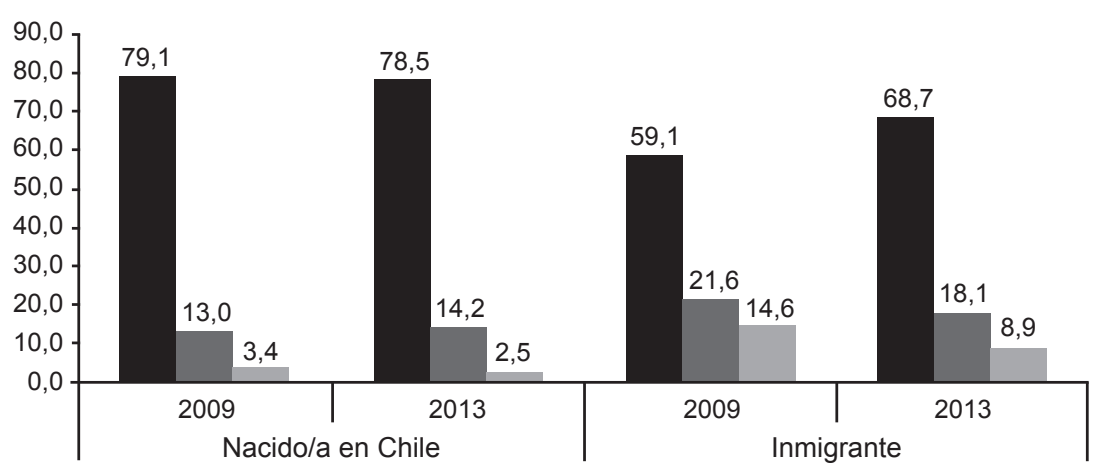

FONASA Isapre Ninguno

Fuente: CASEN (2013)

${ }^{1}$ EI FONDO NACIONAL DE SALUD es la cobertura pública de salud que funciona a partir de una lógica de co-pago.

${ }^{2}$ Las ISAPRES son las coberturas privadas de salud. Estas empresas funcionan también a través de copagos y las personas pueden adscribirse a ellas, si no están en el fondo público.. 
Teniendo en cuenta estos datos, pese a no ser un porcentaje importante de la población residente, la institucionalidad pública requiere dar atención a estos nuevos/as ciudadanos/as residentes que demandan algunos servicios sanitarios. Ante ello, una de las actuaciones que marca un punto de inflexión en el tema migratorio en Chile, tiene que ver con el instructivo presidencial número 9 del año 2008, que asegura la atención básica de salud y de educación, independiente de la procedencia nacional. No obstante, el principal escollo con que se encuentra la población inmigrada en Chile es la documentación exigida para residir en el país de forma legal y el marco restrictivo que tiene la actual Ley de Extranjería. Esto genera que existe una población flotante de personas en situación irregular que no puede acceder a la oferta programática social. Por ello, actualmente se está debatiendo en diversos círculos políticos y sociales, la posibilidad de una nueva política migratoria que esté más acorde con una perspectiva de derechos.

\section{METODOLOGÍA}

En términos metodológicos el estudio se realizó bajo un enfoque mixto: tomando aspectos cuantitativos para el análisis de información de base de datos secundarias, y también interpretativo-hermenéutico, a partir de la aplicación de diversas técnicas de metodología cualitativa. El objetivo de este modelo fue lograr un análisis transversal del fenómeno de la inmigración y su nivel de acceso a diveras áreas sociales, entre ellas los servicios de salud, situando esta perspectiva bajo la luz de los condicionantes económicos, legales, sociales y políticos que inciden en la inserción de las personas inmigrantes. Para llevar a cabo este estudio se recurrió a un conjunto de fuentes de información, tanto primarias como secundarias. Las fuentes primarias fueron abordadas a través de 45 entrevistas y 5 focus group, llevados a cabo en cuatro regiones de Chile (AricaParinacota, Antofagasta, Región Metropolitana y Magallanes) y las fuentes secundarias a través del análisis estadístico a nivel nacional y regional sobre población migrante del Ministerio del 
Interior (DEM); Ministerio de Salud; la encuesta CASEN 2013, y los datos de la ex Ficha de Protección Social ${ }^{3}$.

\section{LÍNEAS TEÓRICAS: INCLUSIÓN Y PERSPECTIVA DE DERECHOS}

Este estudio abordó la temática de la población inmigrada en Chile a partir de un abordaje reflexivo del binomio "exclusión/ inclusión social" y de las diferentes estrategias que se ponen en marcha desde los gobiernos para garantizar una perspectiva de "derechos humanos" de estos colectivos. Cabe señalar que ante los cambios que ha acarreado la globalización como la intensificación de los movimientos trasnacionales, se pueden visualizar también a nivel local nuevos desequilibrios y nuevas desigualdades, que van más allá de la situación socioeconómica, y que puede acarrear otras formas de marginación. De esta manera, siguiendo a Subirats, el concepto de exclusión social sirve para visualizar aquellas situaciones que, más allá de la privación económica, se sufre una privación de la propia idea de ciudadanía, o dicho de otra manera, de los derechos y libertades. (Subirats, 2010). Así, siguiendo a este autor, entenderemos que la inclusión social de un colectivo incluye su participación en diversos ámbitos: a) acceso garantizado a la ciudadanía y a los derechos económicos, políticos y sociales (entre ellos, educación y salud) correspondientes a la misma. b) la conexión y solidez de las redes de reciprocidad social, y c) la participación espacio de la producción económica y del mercado de trabajo (Subirats, 2010). Por tanto, la comprensión de la inclusión/exclusión implicará comprender algunos ejes de diferenciación social sobre los que se vertebran las desigualdades sociales, entre ellos, por ejemplo, la edad, el sexo, la procedencia nacional, el estrato socioeconómico. En este sentido, mujeres, jóvenes, mayores, inmigrados/as, con una situación administrativa regularizada -o no-, o en una situación económica desfavorecida, pueden emerger potencialmente como sectores sociales más susceptibles de padecer ciertos niveles de exclusión.

${ }^{3}$ Esta ficha vigente hasta enero de 2016 medía la situación socioeconómica de las familias a través de la asignación de puntaje que les posibilita o no acceso a ciertos beneficios sociales. Actualmente ha sido cambiada por el Registro Social de Hogares. 
Por tanto, resulta inevitable considerar los temas migratorios desde una perspectiva de Derechos Humanos. El "enfoque de Derechos" constituye un marco conceptual que está basado normativamente en principios y estándares internacionales de Derechos Humanos y operacionalmente dirigido a respetarlos, protegerlos y satisfacerlos. Ahora bien, como señalamos, a pesar de ciertos avances en materia de reconocimiento de derechos o la incorporación en los últimos años de cierta retórica de "derechos" en las políticas sociales aún queda un largo camino por recorrer en la implementación de esta perspectiva. La Declaración Universal de Derechos Humanos, se sintetiza, según Cassese (1993), de la siguiente manera: a) los derechos son inherentes a la naturaleza humana y prescinden de cualquier reconocimiento positivo, es decir, "existen", incluso cuando son negados por el Estado; y b) el orden natural en que se basan los derechos es inmutable, prescindiendo del contexto social de cada individuo. La concepción actual de DDHH recoge también los derechos colectivos de los grupos sociales. Para ser efectivas, las exigencias de respeto de los derechos humanos deben constituir más que una mera declaración, lo que no siempre ocurre. Considerar la matriz de derechos requiere operativizar esta declaración ética, a consideraciones prácticas respecto de cómo se están diseñando e implementando estas políticas públicas, en este caso, que afectan a la población inmigrada. En total relación con la idea de "receptores como sujetos de derecho" aparece como principio rector el "acceso a la justicia", en la medida que no existe un derecho sin que pueda exigirse, de lo contrario sería una mera declaración de principios. Por lo tanto, desde el enfoque de derechos humanos, se requiere esta operativización de las políticas públicas que efectivamente posibiliten mecanismos de reclamo para ser puestos en prácticas en caso de violación de un derecho.

Por otro lado, es preciso reconocer que existen diferentes maneras de intervención estatal en relación con las políticas migratorias (Agrela, 2006). En este sentido, toda intervención plantea un conjunto de desafíos para el Estado cuya función central consiste en garantizar el ejercicio de los derechos ciudadanos. Cabe destacar que el significado alrededor del cual se ha establecido la meta de la gestión de la migración trasnacional para una efectiva inclusión social a nivel internacional es el de "integración", que se ha erigido como "norma" cuyo horizonte es el de evitar una posible 
"ghetización cultural" en ciertos sectores sociales y geográficos y combatir discriminaciones manifiestas, pero también como "promesa" para evitar la exclusión social de este colectivo (Galaz, 2009). Si bien dicho el concepto de integración pretende ser superador de la noción de "asimilación" como eliminación de las diferencias y también de la mera coexistencia sin interacción contenida en algunas propuestas denominadas "multiculturales" (Herrera, 1994) es preciso revisar dicho concepto de integración desde un punto de vista crítico (Gil, 2010). La perspectiva de la integración de la inmigración muchas veces parte del diagnóstico de que ésta se trata de un "problema social" (Galaz y Montenegro, 2015; Santamaría, 2002; Morell Blanch, 2004) en tanto manifestación directa de distintos ejes de diferencia social de las personas con procedencia diversa que residen en un mismo espacio, más que posicionar la idea de inclusión efectiva como un camino para asegurar la igualdad formal en cuanto a derechos y entre las personas nacionales e inmigradas.

Por tanto, la dimensión normativa es un ámbito central para valorar los niveles de participación e inclusión de las personas inmigradas. De esta forma, la reflexión en torno a las políticas públicas tiene que prestar atención a dos aspectos centrales. Por un lado, debe atender al análisis del diseño de la propia política, $y$, por otro, a la capacidad efectiva de resolver los problemas para los cuales ha sido diseñada y las oportunidades que los propios grupos aprecian en las iniciativas que emanan de la institucionalidad pública. Considerando este universo regulativo, se pueden ver los marcos normativos que pueden favorecer o restringir la participación de determinados grupos sociales, como los inmigrados, constituyendo una barrera o un facilitador para el acceso a las garantías, beneficios o prestaciones inscritas en la propia política. En este marco, esta comunicación busca mostrar de qué manera el sistema de salud y sus normativas vigentes poseen barreras y facilitadores para el ejercicio de derechos de la población inmigrada.

\section{RESULTADOS: CARACTERIZACIÓN POBLACIÓN INMIGRADA Y SALUD}

El análisis estadístico desarrollado por la investigación, da cuenta de la implementación de las medidas tomadas en el 
área salud, a partir principalmente del "Programa de Acceso a la Atención de Salud a personas Inmigrantes", aprobado en 2014, para establecimientos de atención primaria de salud, con una cobertura inicial de once comunas, cinco de las cuales constituyen planes piloto. Este programa se lleva ejecutando en centros de atención primaria de las comunas en que el fenómeno inmigratorio está más presente, esperando su expansión a un mayor número de comunas de manera incremental. Por otro lado, a nivel ministerial, se estableció un equipo sectorial asesor de Salud de Inmigrantes, que cuenta con representación de la Subsecretaria de Salud Pública, Subsecretaria de Redes Asistenciales, FONASA y la Superintendencia de Salud. El objetivo a largo plazo es implementar una Política de Salud que considere a la población migrante.

Como señalamos a nivel nacional queda en evidencia que la población inmigrada está más vinculada al sistema público de FONASA. Al analizar la situación por regiones, se reitera esta tendencia, salvo en dos casos que comentaremos a continuación. En la región de Arica y Parinacota, la mayoría de inmigrantes $(70,6 \%)$ cotizan en el sistema previsional FONASA. No obstante, lo preocupante en esta zona del país, es la cifra de extranjeros/as que no está bajo ningún sistema de cotización previsional $(24,8 \%)$, lo que quiere decir que casi 1 de cada 4 inmigrantes de la región no cotiza para ningún sistema. Este dato es un reflejo de que existe aún un número elevado de personas inmigrados/as que por diferentes motivos, como pueden ser, desconocimiento, desinterés o desprotección por parte de las empresas en las que trabajan, o bien por su nivel de transitoriedad al ser una zona limítrofe con otros países (Perú y Bolivia), en caso de problema de salud, no estarían dentro de ningún sistema de cotización previsional. También puede deberse a la presencia de personas inmigrantes que están amparadas por el tratado de MERCOSUR que para mantenerse en el país no requieren una visa sujeta a contrato. Esto lleva, según los datos cualitativos de la zona, a que un sector de la población boliviana trabaje sin contrato y por tanto, sin protección de salud. Asimismo, puede deberse a la alta movilidad existente en la zona. 


\section{Gráfico 3. Distribución de la población nacida en Chile e inmigrante según sistema de cotización previsional Arica Parinacota}

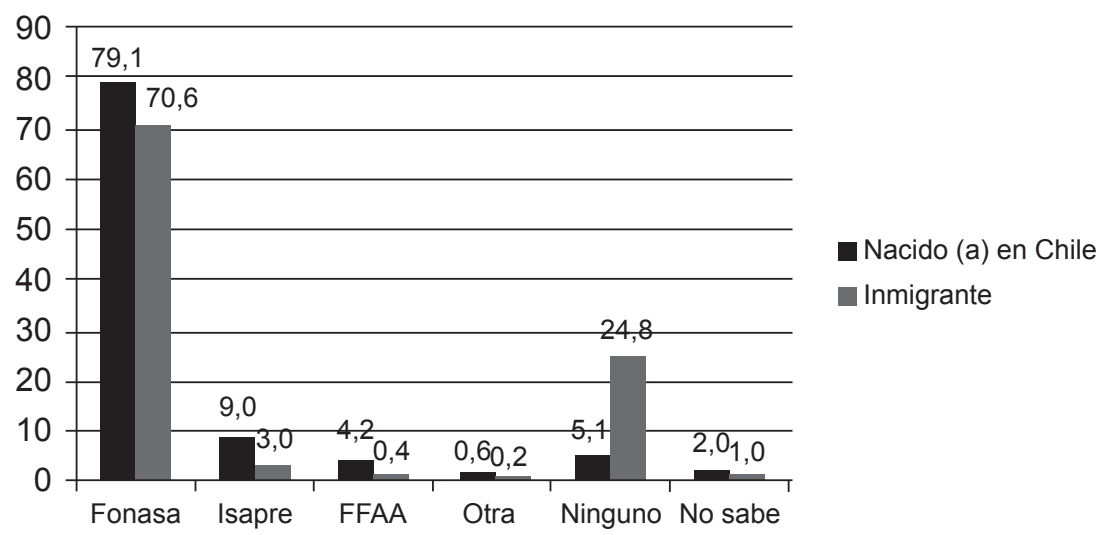

Fuente: CASEN (2013)

También resulta importante los datos que arroja la región de Antofagasta, donde los/las inmigrados/as prefieren el sistema privado de salud por encima del sistema público, incluso lo contratan en mayor proporción que los/las chilenos/as. Esto puede deberse al nivel de ingresos de algunos grupos de inmigrados/ as que trabajan en sectores de la minería en esa zona. Por otro lado, debe considerarse que las personas en situación irregular no están siendo reflejadas en estas estadísticas y por tanto, no se evidencia su falta de cobertura sanitaria. 
Gráfico 4. Distribución de la población nacida en Chile e inmigrante según sistema de cotización previsional Antofagasta

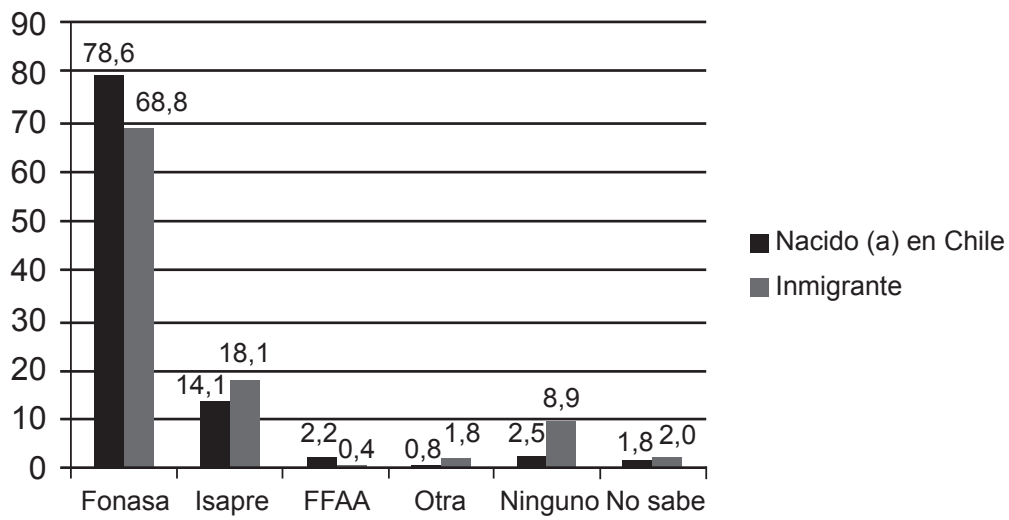

Fuente: CASEN (2013)

\section{Ejercicio de derechos y nudos críticos en salud}

Un primer punto a relevar es que es el ámbito de salud y de educación donde emerge en el año 2007 en Chile, las primeras preocupaciones sectoriales respecto del tema migratorio. Por ello, en este ámbito es que en general existe conciencia acerca de la presencia de los/as inmigrantes en el país y la demanda que eso implica para los diversos servicios y particularmente para los de salud. Esto es un punto importante ya que el "reconocimiento de su presencia" marca un primer paso en orden a promover su acceso y permanencia en el sistema de salud. De hecho en los últimos años, el ministerio de Salud ha desarrollado un conjunto de decretos y normativas acerca de la atención a la población inmigrada los que han sido asumidos medianamente a nivel regional y comunal. En términos concretos, el Ministerio de Salud cuenta con el ordinario A 14 $N^{\circ} 3229$ de junio de 2008, que señala que garantiza la atención para los/as niños/as menores de 18 años al margen de su condición migratoria, para las mujeres embarazadas e incluso 
para "los extranjeros que se encuentran en Chile sin haber regularizado su situación migratoria", quienes tendrán derecho a la atención de urgencia. Del mismo modo, está el Ordinario Gab. Pres. №009 de septiembre de 2008, que también imparte instrucciones a todas las reparticiones del Estado en relación a la apertura del sistema a personas extranjeras. En el cuadro siguiente, se sintetizan los principales derechos enunciados en normativas, decretos y ordinarios en salud:

\section{Tabla 2: Derechos Garantizados en salud}

\begin{tabular}{|c|c|}
\hline \multirow{5}{*}{ SALUD } & $\begin{array}{c}\text { Los/as inmigrados/as regulares tienen acceso a todos los servicios de } \\
\text { salud, incluidas las prestaciones GES en igualdad de condiciones que los } \\
\text { nacionales. Los/as inmigrados/as en situación irregular sólo tienen derecho } \\
\text { a la atención gratuita en los servicios de urgencia de la red asistencial. } \\
\text { Los/as niños/as y adolescentes menores de } 18 \text { años al margen de su situa- } \\
\text { ción migratoria y la de sus padres, tienen derecho a ser atendidos/as en los } \\
\text { establecimientos de salud primaria en igualdad de condiciones. } \\
\text { Las mujeres embarazadas tienen derecho a ser atendidas, en forma inde- } \\
\text { pendiente a su situación migratoria, en los centros de salud que correspon- } \\
\text { dan a su domicilio y pueden, en forma adicional, solicitar una visa tempora- } \\
\text { ria especial solo presentando su certificado de embarazo. }\end{array}$ \\
\hline
\end{tabular}

Sin embargo, pese a estas disposiciones, en la práctica todavía se visualizan algunos nudos críticos que afectan la satisfacción de necesidades de los colectivos inmigrados. Así lo vemos en la siguiente tabla: 


\section{Tabla 3. Nudos críticos en salud}

\begin{tabular}{|c|c|}
\hline \multirow{1}{*}{ SALUD } & $\begin{array}{c}\text { Sistema público como posibilidad única por gran parte de comunidad } \\
\text { inmigrada ante el rechazo de las isapres para aceptar entre sus afiliados } \\
\text { el ingreso de personas inmigradas sin visa definitiva }\end{array}$ \\
\cline { 2 - 3 } & $\begin{array}{c}\text { Diferencias culturales que se presentan en la atención de personas } \\
\text { inmigradas y autóctonas, en relación a los sistemas de salud de origen } \\
\text { y el chileno. }\end{array}$ \\
\cline { 2 - 3 } & $\begin{array}{c}\text { Diferencias en patrones de natalidad (aumento de atención de personas } \\
\text { inmigradas embarazadas-prejuicios sociales sobre coberturas a nivel de } \\
\text { la comunidad autóctona) }\end{array}$ \\
\cline { 2 - 3 } & $\begin{array}{c}\text { Prevalencia de algunas prácticas de riesgo en un sector minoritario de } \\
\text { población migrada }\end{array}$ \\
\cline { 2 - 3 } & $\begin{array}{c}\text { Dificultad básica en cuanto a Políticas Públicas: } \\
\text { Atención supeditada a un número de residencia o RUT. Esto afecta princi- } \\
\text { palmente a las personas no regulares }\end{array}$ \\
\hline
\end{tabular}

Fuente: elaboración propia

Ante estos nudos críticos, se realizó un análisis de la oferta programática disponible desde el ámbito de salud para la población inmigrada. Se seleccionaron para el análisis aquellos programas sociales vinculados al Ministerio de Salud relacionados con las necesidades de los colectivos de inmigrantes, expresadas a través del trabajo cualitativo. A nivel general se puede señalar que existe un desconocimiento por parte de las personas inmigradas de cuáles son los programas a los que pueden acceder incluso al estar en situación de irregularidad como sería el caso de las mujeres embarazadas y los niños/as que asisten al sistema escolar, lo que implicaría una revisión de los mecanismos de difusión de dicha oferta programática, así como los medios de comunicación utilizados para divulgar los servicios a los que se puede acceder.

Más allá del derecho de acceso a la atención en salud entre la población inmigrada y de los obstáculos que aparecen formal e informalmente, es interesante detenerse en las diferencias de uso de los programas de salud debido a las desigualdades en función del estrato socioeconómico. Los estratos socioeconómicamente desfavorecidos tienden a tener menor acceso a servicios preventivos, mayor tiempo de espera para acceder a 
atención especializada y recurren en mayor medida a las urgencias (Regidor, et al., 2002; Palanca, 2002). En el caso de las prestaciones de salud, la disponibilidad temporal y/o por la existencia de co-pagos para determinadas prestaciones limitan el acceso de la población inmigrada (por ejemplo, en la atención dental, en el tratamiento de enfermedades crónicas y leves, en los exámenes y procedimientos médicos menores). Cabe destacar que estas dificultades también las presentan los sectores sociales chilenos ubicados en los quintiles de menos ingresos. Esto apunta a la existencia de condicionantes externos que van más allá de la voluntad de los/las pacientes, lo que genera diferentes oportunidades de acceso a la atención en salud en función del nivel de renta y/o educativo de las personas inmigradas.

El efecto de los programas en materia de salud es, en general, positivo: concretamente, se observan incrementos significativos de los controles preventivos de salud, el acceso a los servicios de salud y la utilización de los servicios ambulatorios. Por tanto, se puede visualizar claramente un acceso fácil a un sector específico de la población inmigrada constituida por las mujeres embarazadas y niños/as, que son a los que se ha focalizado las acciones de inclusión en salud, pero aún existe un amplio grupo de población migrante que queda excluido de las prestaciones de salud como sería el caso por ejemplo de la población adulta mayor que representa patologías crónicas o es el caso de hombres adultos. Las diferencias en el acceso de los programa públicos se explican por los criterios de focalización que han implementado los diversos organismos gubernamentales. Si bien, en algunos casos no existen restricciones por criterios de focalización, la exclusión se produce debido a la documentación solicitada.

Para lograr avances en esta materia se debiera tener presente que la mayor parte de los programa sociales, actualmente responden a una perspectiva de inclusión, que opera desde una mirada monocultural -que busca asimilar la población inmigrada a la población local- exigiendo para ello el cumplimiento de los mismos requisitos, incluso sin considerar las diferencias culturales y administrativas del país de origen. Quizás tomar el incipiente esfuerzo institucional que existe respecto de algunos sectores 
indígenas como modelo para sugerir políticas similares a este colectivo, sobre todo en algunas zonas del sur y norte del país.

\section{Obstaculizadores y Facilitadores en el ámbito de salud}

El análisis cualitativo desarrollado, permitió extraer algunos obstaculizadores y facilitadores dentro del sistema de salud que afecta la inclusión efectiva de la población inmigrada. Entre los principales obstaculizadores, se consideran cuatro grandes temáticas:

a) Crítica respecto de la salud pública que se relacionan con el nivel de cobertura y nivel de recursos de esta área para atender a toda la población residente. Más concretamente, se aprecia largos tiempos de espera para atención primaria y especialmente secundaria (operaciones, especialidades), falta de profesionales, visitas médicas de rápida atención y/o programación a largo plazo. Sin embargo, esta es una situación compartida con la comunidad chilena, lo que dice relación con la capacidad del sistema de salud en general. En ese sentido, los/as inmigrantes comparten la misma crítica que la población chilena en cuanto a la forma en que algunos servicios de salud son gestionados y atendidos. Por ejemplo, en algunos relatos se plantea como obstaculizador al acceso en la región de Antofagasta, en el norte del país, las malas condiciones de las instalaciones del Hospital público y una escasez de centros básicos a nivel de ciudad. Esto lleva a que efectivamente en algunos centros de salud se concentre por zonas de residencia, la población migrada, con la consecuente renuencia de la población local.

b) Acceso diferenciado de la población inmigrada a los distintos niveles de salud (primaria, secundaria y hospitales). En particular, existen dificultades para el acceso equitativo a la atención secundaria de salud y de enfermedades crónicas porque el acceso está mediado por la afiliación a FONASA en alguno de sus tramos, para lo cual es evidente que se debe tener un número de cédula nacional (RUT), a lo cual se accede únicamente a través de la condición migratoria regular. Por tanto, todas las personas que están en situación irregular no pueden acceder 
a esta cédula y a su vez, no pueden ser afiliadas al sistema público de salud, quedando excluidas de la protección social en este sentido. Por otra parte, el hecho de estar afiliado a FONASA no asegura la atención, ya que parte de las atenciones no provistas por el sistema de salud como exámenes o atenciones específicas médicas al ser un sistema de co-pago, parte de la población inmigrada debido a sus condiciones socioeconómicas, no pueden pagar prestaciones y servicios, sobre todo las de larga duración (enfermedades crónicas y hospitalizaciones), lo que lleva a un acceso diferenciado. Evidentemente esto supone una clara distinción entre la población inmigrada de acuerdo al estatus que posea: por un lado, quienes están en condición regular acceden a los mismos derechos que la población nacional, incluso a las prestaciones GES, mientras que quienes están en condición irregular presentan obstáculos muchas veces insalvables para la atención en salud secundaria.

Un tema aparte es el tratamiento de enfermedades crónicas: según el estudio, gran parte de la población inmigrada residente acude principalmente ante emergencias a los centros de salud (principalmente por el co-pago o por la situación migratoria, por la falta de tiempo libre en los horarios de atención y también por consideraciones culturales sobre la salud/ enfermedad), por tanto no acude a los centros hasta un grado de avance de algunas enfermedades.

"En el ámbito de la salud quizás haya un problema bien grave. Con la atención secundaria, porque en general en primaria, independiente que estén regulares o irregulares, se supone que acceden, pero hemos visto casos bien complejos, sobre todo en mujeres que no pueden acceder" (Extracto entrevista Dirección Salud. Región Metropolitana, 2015).

"Y ahora mismo tenemos un caso de una señora que falleció la semana pasada, el viernes pasado. Es irregular pero recibió la atención de salud, pero tenía un cuadro asmático crónico, probablemente no medicado en los últimos meses". (Extracto entrevista. Consulado República Dominicana. 2015).

Aunque este tipo de situaciones pueden ser excepcionales, se deriva como problema de la política pública en tanto ponen 
en evidencia una falencia respecto de la atención de enfermedades de otro tipo, como por ejemplo los accidentes laborales o profesionales que se derivan de obras o faenas en las cuales trabaja parte de la población en condición irregular, e incluso las enfermedades crónicas.

c) Falta de acceso de la población inmigrada por falta de información del cuerpo funcionario. Ante la exigencia normativa de una cédula nacional de identidad o de FONASA para la atención, se pueden reseñar algunos casos de vulneración de derechos por parte de funcionarios/as, toda vez que no se reconoce el acceso de algunas personas protegidas formalmente pese a su condición irregular a los servicios de atención, al no haber mediado un pago o la posesión de un RUT válido. Esto, algunos lo explican, por la falta de información sobre las directrices generales del Ministerio que posibilita a ciertos colectivos a la atención gratuita y sin RUT, que ha llevado a que algunos casos de vulneración de derechos se vuelvan mediáticos ya que ha conllevado la muerte de algunas personas inmigradas o de riesgo vital de mujeres embarazadas.

"Lo que hemos detectado es que en algunos hospitales hay más desconocimiento. Nos ha pasado gestionar reclamos respecto a cobros a mujeres embarazadas" (Exctrato de entrevista. Profesional Municipalidad de Estación Central. Chile. 2015).

"En caso de urgencias sí lo van a atender, pero la persona tiene que pagar los exámenes por ejemplo y después de atenderse todavía le hacen firmar un pagaré y tendrá que pagarlo". (Extracto consulado Haití. Chile. 2015).

d) Problemáticas asociadas a la atención de los/as funcionarios/as de salud. Existe en algunos casos una visión crítica del trato de funcionarios/as hacia la población inmigrada, además de dificultades de entendimiento cultural e incluso idiomático. En los relatos cualitativos se puede visualizar una crítica al "trato" que brindan alguno/as funcionarios/as a la población inmigrada -principalmente administrativos y personal médico y de cuidados- que se basa en estereotipos y rumores respecto de los colectivos inmigrados, generando algunas situaciones discriminatorias que vulneran los 
derechos de personas extranjeras. A lo anterior también se suma una dificultad que afecta a algunos grupos en particular, como por ejemplo haitianos/as, cuyo idioma muchas veces se transforma en una barrera adicional de acceso a los servicios de salud (dado que por lo general tanto el personal técnico como profesional de los servicios de salud no habla otro idioma). En tales casos suelen ser los/as niños/as quienes ejercen laborales de traductores de traducción aunque no con la exactitud necesaria para procesos asociados a la medicina, lo que puede constituir un riesgo para la salud de las personas.

En cuanto a los facilitadores para la accesibilidad a salud, se pueden señalar cuatro grandes temáticas:

a) Reconocimiento institucional explícito acerca de la presencia de los/as inmigrados/as en Chile y sus necesidades en el área de salud. Evidentemente, los decretos emanados al respecto constituyen un primer punto que da cuenta de una preocupación que trasciende el campo de acción de un ministerio, situándose como un tema nacional. Por otro lado, tanto en las organizaciones de inmigrados/as como en las ONG que trabajan con la población, se reconoce como un facilitador el hecho de tener acceso quienes disponen de FONASA al menos al sistema de salud primaria. Por otro lado, también se destaca la atención que recibe la población inmigrada por parte de algunos/as profesionales de la salud más sensibilizados con la temática y quienes han promovido cambios a nivel institucional para acercar el aparato de salud a este colectivo. Muchas veces esta disposición positiva de profesionales se relaciona con la disposición positiva de los municipios donde se insertan estos centros de salud y los propios discursos pro-inmigración que existen a nivel de gobierno local. En ese sentido, se coloca como ejemplo los dispositivos temporales que se colocan en espacios públicos cercanos a los espacios de vivienda de la población inmigrada y en horarios de fines de semana para atender a esta población (estos dispositivos nacen en algunos centros urbanos de la Región metropolitana, más sensibles a la temática y ante la evidencia de que las personas no acudían a los centros de atención familiar). 
"Se puede criticar muchas cosas pero también hay cuerpo médico comprometido. Como responsable de la unidad de migrantes, me han llamado para decirme: he atendido a esta persona, dígame qué puedo hacer yo como médico para que entre a la cobertura de AUGE" (Extracto de entrevista encargada municipal, Quilicura, Chile, 2015).

b) Desarrollo de algunos mecanismos informales que facilitan el acceso de la población inmigrante no regular. Esto hace referencia a algunas medidas que desde la preocupación específica de algunos/as profesionales se desarrollan esporádicamente para facilitar la atención de las personas inmigrantes no regulares, aunque no sean conductos formales, como el hecho de colocar un RUT ficticio en la ficha para poder ingresarle y posibilitarle el servicio, o colocar el RUT propio, o pasarlo en "situación de indigencia" para no hacer efectivo los cobros asociados.

"Ahora el tema del carnet o de Fonasa, hay algunas triquiñuelas que se hacen... algunos funcionarios, con la ficha electrónica. Ponen el nombre del funcionario y por ahí les abren las puertas a los medicamentos. $A$ veces es un tema que se arregla al estilo chileno" (Extracto entrevista coordinadora ONG. Chile. 2015)

Si bien estas medidas son enunciadas como prácticas aisladas y reconocidas como un facilitador, lo que trasciende es que existen muchas situaciones que quedan al arbitrio ya sea de los/ as funcionarios/as o de la propia voluntad política de las autoridades. De ahí la necesidad e importancia de diseñar y consolidar políticas y protocolos de atención para los profesionales de la salud que vayan en beneficio de la población inmigrada.

c) Rol de entidades de la sociedad civil, algunos consulados y oficinas de inmigración como "agencias mediadoras". Aquí se consideran las intermediaciones que realizan en el ámbito sanitario diversas ONG y también algunas oficinas para los/las inmigrados/as y algunos consulados específicos que facilitan su acceso a los servicios de salud. El apoyo puede ser desde entrega de información, acompañamiento a trámites específicos, traducción y apoyo a comprensión de instructivos y normativas, hasta pagos directos por prestaciones en 
casos con más vulnerabilidad social. En este último caso, algunas entidades asumen el costo ante personas que no pueden asumir la deuda o el apoyo ante enfermedades no cubiertas por el sistema de salud.

d) Existencia del comité de "integración fronterizo" entre Perú y Chile que opera a nivel de servicio de salud de Arica y el de Tacna. Aunque es una iniciativa focalizada territorialmente en la zona norte del país, implica la coordinación en diversos niveles de atención a la población de ambos países y que coincide en ser de ascendencia indígena y además inmigrada. Una de sus áreas de trabajo más desarrollada es la de salud. Por otro lado, en Tacna Perú funciona un Hospital Solidario que ha ayudado a que muchas personas chilenas, peruanas y bolivianas- a precios bajos sean atendidas en ese servicio y desentrampe al hospital regional de Arica.

"En algunos temas críticos como la tuberculosis... hemos hecho intercambios de salud...este tipo de integraciones se hace, hay otros convenios como traslado de medicamentos que a veces se requieren, aunque es difícil tener protocolos tan claros. Generalmente se hacen planes de colaboración conjunta, a nivel de comunidades, de intercambio médico, trabajo en fronteras...en el traslado de enfermos o fallecidos, por situaciones de desastre... aunque no hay acuerdo para la atención de personas". (Extracto entrevista profesional. Gob. Salud. Arica y Parinacota, Chile, 2015)

\section{CIERRE CONCLUSIVO}

A partir del análisis desarrollado, resulta evidente que quedan vacíos en los niveles de accesibilidad que tiene la oferta programática de salud, con tal de dar protección universal a las personas que viven en Chile, entre ellas, la comunidad inmigrada. Por otra parte, ante el hándicap desde el que se inician en el proceso de inclusión social, también en el estudio se constató la necesidad de generar programas específicos que considere a este sector, visualizando las desventajas que poseen para situarse en igualdad de condiciones que el resto de la población 
nacional. Esto implicaría desarrollar medidas y prescripciones que considere variables que no han sido consideradas y que tomen en cuenta la diversidad sociocultural actual del territorio.

Sin duda, esto implica re-pensar algunas dimensiones a priori, como es el binomio ciudadanía/inclusión (Subirats, 2010): ¿a quiénes se considera como "ciudadanos/as" dentro de un territorio diverso?; y ¿quiénes pueden ser considerados/as como efectivos depositadores de derechos civiles y sociales? La configuración social para la inclusión será diferente si consideramos la ciudadanía al margen de la nacionalidad, o bien si se establecen accesos restringidos y escalados de ciudadanía, y por último, si se consideran niveles de participación enunciativas, consultivas o de efectivo empoderamiento social. Las barreras burocráticas que aún existen, tienen relación con este tipo de preguntas y afecta directamente al sistema de salud chileno actual.

Las personas inmigradas se enfrentan con obstaculizadores concretas a nivel sanitario para su efectiva inclusión social no sólo derivadas de aprehensiones socioculturales y de un cierto rechazo de la comunidad chilena de recepción o del funcionariado que les atiende, sino que también a partir de dificultades impuestas desde el mismo Estado: legislativa, administrativas, de gestión, y de falta de una efectiva "acogida" inicial y traspaso de información sobre la inserción a la cobertura sanitaria y de la oferta programática disponible. Para este colectivo aún la adquisición de una documentación regular (visa, RUT, certificación FONASA) sigue siendo una gran problemática que solventar y en la que se centra la instalación en el nuevo contexto de vida, lo que condiciona a su vez su participación en otras áreas de protección social más general. Esto lleva incluso a un círculo vicioso: que un sector minoritario en situación de vulnerabilidad dentro del grupo de personas inmigradas -como son aquellas en situación irregular y sin posibilidad de acceder a RUT- quede al margen de beneficios directos precisamente porque no disponen de los requisitos exigidos para poder acceder a nivel de visados. De esta manera, aquel sector que más requiere protección social dentro de todo el colectivo de población inmigrada residente, precisamente queda imposibilitado de poder llegar a esta oferta. De ahí que se requiera una operativización de las políticas públicas que efectivamente posibiliten mecanismos de reclamo para ser puestos en prácticas en caso de violación de 
los derechos que se enuncian actualmente como posición ética. Se requiere una dimensión procesal, es decir mecanismos institucionales que permitan traducir esos acuerdos normativos de derechos humanos en instrumentos de política social y de intervención directa, y finalmente una dimensión de contenidos relativos a la protección social, que oriente acciones concretas respecto de cierto sector de la población inmigrada en general y en particular, aquella que se ubica en condiciones de vulnerabilidad. De lo contrario, se seguirán produciendo situaciones en que queda al arbitrio de los/las funcionarios/as el acceso y goce de los beneficios sanitarios.

Existe una ausencia de esta lógica de derechos humanos como eje transversal en el diseño de los programas públicos. Si bien la mayoría de los programas, salvo unas cuantas excepciones, no se desarrollan desde una visión nacionalista y exclusivista respecto de la exigencia de ser chileno/a para optar a beneficios; un porcentaje considerable plantea requisitos que dejan fuera a un sector de la población inmigrada, como es aquella en situación irregular (exigencia de Fonasa). Por otro lado, gran parte de los programas establecen requisitos que dan por sentado una trayectoria de vida en el país que explicita que en su diseño no fueron pensadas personas que pudieran llevar pocos años de residencia. De esta manera, las personas inmigrantes para acceder a estos beneficios requieren al menos tener un tiempo de estancia en el país y haber cruzado por diversos trámites administrativos previos (tener RUT vigente, haber llenado la Ficha de Protección Social, tener residencia definitiva, tener FONASA, entre otros requisitos).

Desde una perspectiva técnica, la aplicación de una nueva ley que incorpore cambios profundos orientados a instalar en Chile un enfoque de inclusión intercultural y de reconocimiento de la diversidad, generará efectivamente un mayor impacto, pero ello conllevará la articulación de procedimientos sistemáticos, fiables y disponibles para la obtención y gestión de visados. Por ello, se deben mejorar los sistemas de registro de la información situados en diferentes estadios temporales del proceso mismo de inmigración.

Por otro lado, en el estudio se visualizó cómo generalmente los diversos actores del Estado consideran la inclusión de este 
sector casi exclusivamente desde la posibilidad de "acceso". Sin embargo, a partir de la experiencia analizada, el acceso básico a la salud no es suficiente para garantizar efectivamente el goce de los derechos ni muchos menos, favorecer la participación de este sector en igualdad de condiciones que la población chilena. Se requieren además procesos de gestión interna que posibilite, por ejemplo, que la información llegue a este colectivo; que se establezcan mecanismos administrativos que favorezcan su relación y ubicación en este nuevo contexto social. A esto se suma favorecer una transformación cultural a través de acciones que promueva una aceptación social de la expresión de la diversidad sociocultural. Posibilitar la accesibilidad del Estado a la población inmigrada, implica no sólo combatir las barreras burocráticas discutidas en este estudio, sino también adecuarse a nivel lingüístico, considerar aspectos como el estatus legal de las personas inmigradas, el género, la edad y la nacionalidad de procedencia de éstas.

De esta manera, promover la inclusión social apuntaría no sólo a tomar como centro la transferencia de recursos económicos, sino transferencias sociales, políticas, culturales que fortalezcan la autonomía y la participación de los sujetos inmigrantes. Se trataría de construir las condiciones para una ciudadanía basada en el respeto de los derechos individuales y sociales, considerando la diversidad sociocultural actual.

\section{BIBLIOGRAFÍA}

Aday L.A. y Andersen R.M. (1974). A framework for the study of access to medical care. Health Serv Res. 1974; 9: 208-20

Aday L.A. y Andersen R.M. (1993). Theoretical framework for the study of access to medical care. Health Serv Res. 1993; 208-22.

Agar Corbinos, L. (2012). Migraciones posmodernas en Chile: Reflexiones sobre cohesión social y pluralidad cultural, Palabra (12) Agosto de 2010 - Julio de 2011.

Agar Corbinos, L. y Saffie Guevara, N. (2010). Migrantes en Chile: políticas públicas en salud, cohesión social e interculturalidad; en Agar Corbinos (Coord) Migraciones, salud y globalización: entrelazando miradas. Santiago de Chile: Biplano. 
Agrela, Belén (2006). Análisis antropológico de las políticas sociales dirigidas a la población inmigrante. Tesis doctoral dirigida por Carmen Gregorio Gil, Universidad de Granada. Disponible en: http://ldei.ugr.es/cddi/fichates.php?id=3\&tabla=tesis [Acceso: 19 de abril de 2008].

Burón A., Cots F., García O., Vall O., Castells X. 2014). Hospital emergency department utilisation rates among the immigrant population in Barcelona, Spain. BMC Health Serv Res. 2008; 8: 51.

Carmona R., Alcázar-Alcázar R., Sarría-Santamera A., Regidor E. (2014). Frecuentación de las consultas de medicina general y especializada por población inmigrante y autóctona: una revisión sistemática. Rev Esp Salud Publica. 2014; 88: 135-55.

Cassese, A. (1993). Los derechos humanos en el mundo contemporáneo. Barcelona: Ariel.

Daponte Codina A., Bolívar Muñoz J., García Calvente M.M. (2008). Las desigualdades sociales en salud. 1a ed. Granada: Escuela Andaluza de Salud Pública.

Esping-andersen, G. (1993). Los tres mundo del Estado de Bienestar. Valencia: Edicions Alfons El Magnànim.

Esping-andersen, G. (1996). "Después de la Edad de Oro: el futuro del Estado Benefactor en el nuevo orden mundial". Revista Desarrollo Económico, Instituto de Desarrollo Económico y Social, 36 (142), 523-553.

Galaz, C. y Montenegro, C. (2015). Gubernamentalidad y relaciones de inclusión/exclusión: los dispositivos de intervención social dirigidos a mujeres inmigradas en España. Universitas Psychologica, 14(5).

Galaz, C. (2016). Intervención social con mujeres inmigradas: Reconocimiento, incorporación sociocultural e igualdad de oportunidades para la inclusión. U-Inicia. Universidad de Chile.

Galaz, C. (2009). Las relaciones de cooperación y exclusión social. Un estudio sobre la alteridad. Barcelona: Universidad Autónoma de Barcelona.

Geddes, Andrew (2006). Inmigración y Estado de Bienestar en Europa, Zona Abierta, 116-117, 171-190. 
Geddes, Andrew (2008). Immigration and European Integration: Beyond Fortress Europe? Manchester: University Press, Manchester.

Gómez-Camelo, D. (2005). Análisis comparado de los sistemas de salud de la región Andina y el Caribe. Rev salud pública, 7(3), 305-316

Gil Araújo, S. (2010). Las argucias de la Integración: Políticas migratorias, construcción nacional y cuestión social. Madrid: lepala.

Herrera, Encarna (1994): Reflexiones en torno al concepto de integración en la sociología de la inmigración, Papers, 43, 71-76.

Honneth, A. (1997). La lucha por el reconocimiento: por una gramática moral de los conflictos sociales. Barcelona: Editorial Crítica.

Honneth, A. (2007). Reificación. Un estudio en la teoría del reconocimiento. Madrid: Katz.

Jiménez-Rubio, D., Hernández-Quevedo, C. (2012). Inequalities in the use of health services between immigrants and the native population in Spain: what is driving the differences? Eur J Health Econ. 2011; 12: 17-28.

Mladovsky, P. (2007). Reseach Note: Migration and Health in the EU. Brussels: European Commission.

Morell Blanch, A. (2004). La inmigración como problema: un análisis de las prácticas discursivas de la población autóctona, $\mathrm{Pa}-$ pers, 74, 175-201.

Naciones Unidas (2000). Derechos Humanos de los Migrantes. Resolución 2000/48. Nueva York, Oficina del Alto Comisionado de las Naciones Unidas para los Derechos Humanos. Presidencia de la República de Chile (2008) Ordinario Gab. Pres. N009 de septiembre de 2008.

Organización Mundial de la salud. (2016, Junio 15). http://www. who.int.es. Retrieved from http://www.who.int/features/ ga/28/es/

Rué, M., Cabré, X., Soler, J., Bosch, A., Almirall, M., Serna, M.C. (2008). Emergency hospital services utilization in Lleida (Spain): A cross-sectional study of immigrant and Spanish-born populations. BMC Health Serv Res. 2008; 8. 
Rodríguez Álvarez, E., Lanborena Elordui, N., Pereda Riguera, C., Rodríguez Rodríguez, A. (2008). Impacto en la utilización de los servicios sanitarios de las variables sociodemográficas, estilos de vida y autovaloración de la salud por parte de los colectivos de inmigrantes del País Vasco, 2005. Rev Esp Salud Publica. 2008; 82: 209-20

Ramos, J. M., García R., Prieto M.A., March J.C. (2001). Problemas y propuestas de mejora en la atención sanitaria a los inmigrantes económicos. Gac Sanit. 2001; 15: 320-6.

Terraza-Núñez R., Toledo D., Vargas I., Vázquez M.L. Perception of the Ecuadorian population living in Barcelona regarding access to health services. Int J Public Health. 2010; 55: 381-90

Santamaría, E. (2002). La incógnita del extraño. Una aproximación a la signi- ficación sociológica de la "inmigración no comunitaria”. Barcelona: Anthropos.

Subirats, J. (2010). Ciudadanía e Inclusión Social. Madrid: Fundación Esplai. 